\title{
String Representation of Field Correlators in the Dual Abelian Higgs Model
}

HUB-EP-98/34

\author{
Dmitri Antonov ${ }^{* \dagger}$ and Dietmar Ebert ${ }^{\ddagger}$ \\ Institut für Physik, Humboldt-Universität zu Berlin, \\ Invalidenstrasse 110, D-10115, Berlin, Germany
}

\begin{abstract}
By making use of the path integral duality transformation, we derive the string representation for the partition function of an extended Dual Abelian Higgs Model containing gauge fields of external currents of electrically charged particles. By the same method, we obtain the corresponding representations for the generating functionals of gauge field and monopole current correlators. In the case of bilocal correlators, the obtained results are found to be in agreement with the dual Meissner scenario of confinement and with the Stochastic Model of the QCD vacuum.
\end{abstract}

*E-mail address: antonov@pha2.physik.hu-berlin.de

${ }^{\dagger}$ On leave of absence from the Institute of Theoretical and Experimental Physics, B. Cheremushkinskaya 25, 117 218, Moscow, Russia.

${ }^{\ddagger}$ E-mail address: debert@qft2.physik.hu-berlin.de 


\section{Introduction}

The explanation and description of confinement in gauge theories is known to be one of the most fundamental problems of modern Quantum Field Theory (see e.g. [1,2]). In general, by confinement one means the phenomenon of absence in the spectrum of a certain field theory of the physical |in $\rangle$ and |out $\rangle$ states of some particles, whose fields are however present in the fundamental Lagrangian. A natural conjecture here is that due to a linearly rising confining interaction, (anti)quarks cannot exist as free particles, but form colourless bound states of hadrons. The most natural quantity for the description of this phenomenon is the so-called Wilson loop average. In the case of Quantum Chromodynamics (QCD), this object has the following form

$$
\langle W(C)\rangle=\frac{1}{N_{c}}\left\langle\operatorname{tr} \mathrm{P} \exp \left(i g_{\mathrm{QCD}} \oint_{C} A_{\mu} d x_{\mu}\right)\right\rangle,
$$

which is nothing else, but an averaged amplitude of the process of creation, propagation, and annihilation of a quark-antiquark pair. Here, $A_{\mu}$ stands for the matrix-valued vector-potential of the gluonic field, $C$ is a closed contour, along which the quark-antiquark pair propagates, $P$ stands for the path-ordering prescription, and the average on both sides is performed with the QCD action. The interaction potential of a pair of static quark and antiquark can be determined from the Wilson loop average, defined on the rectangular contour $C$, according to the Feynman-Kac formula,

$$
V=-\lim _{T \rightarrow \infty} \frac{1}{T}\langle W(C)\rangle,
$$

where $T$ stands for the size of $C$ in the temporal direction. In particular, for large contours $C$, the exponential dependence of the Wilson loop average on the area of the minimal surface, $\Sigma_{\min }$, encircled by $C$,

$$
\langle W(C)\rangle \rightarrow \mathrm{e}^{-\sigma \cdot\left|\Sigma_{\min .}\right|}
$$

(the so-called area law behaviour of the Wilson loop), yields then the linearly rising confining potential

$$
V_{\text {conf. }}(R)=\sigma R .
$$

Here $\sigma$ stands for the so-called string tension, and $R$ is the relative distance between a quark and an antiquark. This exponential asymptotic dependence of the Wilson loop average on $\left|\Sigma_{\text {min. }}\right|$ is just the essence of the Wilson criterion of confinement [3].

The linearly rising potential (2) admits a simple physical interpretation in terms of a string picture: the gluonic field between a quark and an antiquark is compressed to a tube or a string (the so-called QCD string), whose energy is proportional to its length, and $\sigma$ is the energy of such a string per unit length. This string plays the central role in the Wilson's picture of confinement, since with increasing distance $R$ it stretches and prevents quark and antiquark from moving freely to macroscopic distances. In order to get an idea of numbers, notice that according to the lattice data [4] the distance $R$, at which the Wilson criterion of confinement becomes valid, is of the order of $1.0 \mathrm{fm}$, and the string tension is of the order of $0.2 \mathrm{GeV}^{2}$ (see e.g. [2]). 
As it is well known, all observable dimensional quantities in QCD such as hadron masses, and in particular the string tension, are proportional to the corresponding power of the QCD scale parameter $\Lambda_{\mathrm{QCD}}$ 凹. In particular, one has

$$
\sigma \propto \Lambda_{\mathrm{QCD}}^{2}=\mu^{2} \exp \left[-\frac{16 \pi^{2}}{\left(\frac{11}{3} N_{c}-\frac{2}{3} N_{f}\right) g_{\mathrm{QCD}}^{2}(\mu)}\right],
$$

where $g_{\mathrm{QCD}}(\mu)$ is the QCD coupling constant measured at a scale $\mu$. Eq. (3) means that all the coefficients in the expansion of the string tension in powers of $g_{\mathrm{QCD}}^{2}(\mu)$ vanish. This conclusion tells us that the QCD string has a pure nonperturbative origin, as well as the phenomenon of confinement, which leads to the process of formation of such strings in the QCD vacuum, itself.

The problem of studying the properties of the QCD string is closely related to the problem of finding a string representation of gauge theories possessing a confining phase. In this respect, all methods of derivation of the string effective action from the action of gauge theories are of great importance. Some progress in the solution of this problem has recently been achieved for the case of $Q C D$ in Refs. [5-7] using the so-called Stochastic Vacuum Model (SVM) of the QCD vacuum $[2,8]$.

The idea which lies behind this approach is that important quantities like Green functions can be expressed in terms of the Wilson loop [3,8]. The main strategy of SVM is then not to evaluate the Wilson loop itself, but to express it via gauge-invariant irreducible correlators (the so-called cumulants) of the gauge field strength tensors. Such correlators have been measured in the lattice experiments both at large and small distances [9], which enables one to use them in practical calculations of various physical quantities. In order to express the Wilson loop average (1) via cumulants, one makes use of the non-Abelian Stokes theorem and cumulant expansion (see [2] for a detailed discussion and Refs. therein), which yields

$$
\begin{gathered}
\langle W(C)\rangle=W\left(\Sigma_{\text {min. }}\right) \simeq \\
\simeq \frac{1}{N_{c}} \operatorname{tr} \exp \left(-\frac{g_{\mathrm{QCD}}^{2}}{2} \int_{\Sigma_{\text {min. }}} d \sigma_{\mu \nu}(x) \int_{\Sigma_{\text {min. }}} d \sigma_{\lambda \rho}\left(x^{\prime}\right)\left\langle\left\langle F_{\mu \nu}(x) \Phi\left(x, x^{\prime}\right) F_{\lambda \rho}\left(x^{\prime}\right) \Phi\left(x^{\prime}, x\right)\right\rangle\right\rangle\right),
\end{gathered}
$$

where $\Phi(x, y)$ stands for the parallel transporter factor along the straight line. In the derivation of Eq. (4), one has used the so-called bilocal or Gaussian approximation, according to which all higher cumulants can be disregarded with a good accuracy due to their smallness w.r.t. the bilocal cumulant. This approximation is indeed confirmed by the present lattice data [9].

The bilocal cumulant standing in the exponent on the R.H.S. of Eq. (4) can be parametrized by two renormalization group-invariant coefficient functions $D^{\mathrm{QCD}}$ and $D_{1}^{\mathrm{QCD}}$ as follows [8]

$$
\frac{g_{\mathrm{QCD}}^{2}}{2}\left\langle\left\langle F_{\mu \nu}(x) \Phi\left(x, x^{\prime}\right) F_{\lambda \rho}\left(x^{\prime}\right) \Phi\left(x^{\prime}, x\right)\right\rangle\right\rangle=\frac{\hat{1}_{N_{c} \times N_{c}}}{N_{c}}\left\{\left(\delta_{\mu \lambda} \delta_{\nu \rho}-\delta_{\mu \rho} \delta_{\nu \lambda}\right) D^{\mathrm{QCD}}\left(\left(x-x^{\prime}\right)^{2}\right)+\right.
$$

\footnotetext{
${ }^{1}$ This means that the dimensionless ratios of these quantities, e.g. the ratio of $\sqrt{\sigma}$ to hadron masses, are universal (i.e. $g_{\mathrm{QCD}}$-independent) numbers. The aim of all the nonperturbative phenomenological approaches to QCD (e.g. the so-called Stochastic Vacuum Model, which will be described below) is to calculate these numbers, but not $\Lambda_{\mathrm{QCD}}$ itself.
} 


$$
\left.+\frac{1}{2}\left[\frac{\partial}{\partial x_{\mu}}\left(\left(x-x^{\prime}\right)_{\lambda} \delta_{\nu \rho}-\left(x-x^{\prime}\right)_{\rho} \delta_{\nu \lambda}\right)+\frac{\partial}{\partial x_{\nu}}\left(\left(x-x^{\prime}\right)_{\rho} \delta_{\mu \lambda}-\left(x-x^{\prime}\right)_{\lambda} \delta_{\mu \rho}\right)\right] D_{1}^{\mathrm{QCD}}\left(\left(x-x^{\prime}\right)^{2}\right)\right\} .
$$

Notice, that by construction the term containing the function $D_{1}^{\mathrm{QCD}}$ on the R.H.S. of Eq. (5) yields a perimeter type contribution to the Wilson loop average (4). In particular, the leading perturbative contribution of the one-gluon-exchange diagram to the Wilson loop average is contained in the function $D_{1}^{\mathrm{QCD}}$.

The nonperturbative parts of the functions $D^{\mathrm{QCD}}$ and $D_{1}^{\mathrm{QCD}}$ have been calculated on the lattice in Ref. [9], where it has been shown that at large distances $\left|x-x^{\prime}\right|, D_{1}^{\mathrm{QCD}}\left(\left(x-x^{\prime}\right)^{2}\right) \ll$ $D^{\mathrm{QCD}}\left(\left(x-x^{\prime}\right)^{2}\right)$, and both functions decrease as $\mathrm{e}^{-\left|x-x^{\prime}\right| / T_{g}}$, where $T_{g}$ is the so-called correlation length of the vacuum, $T_{g} \simeq 0.22 \mathrm{fm}$ in the $S U(3)$-case.

The idea realized in Ref. [5] was to consider the quantity $S_{\text {eff. }}=-\ln W\left(\Sigma_{\text {min. }}\right)$ defined by Eqs. (4) and (5) as a starting point for the derivation of the QCD string effective action. Due to the smallness of the ratio $\left(T_{g} / R\right)^{2} \simeq 0.04$, it was then possible to perform an expansion of the nonlocal action $S_{\text {eff. }}$ in powers of $\left(T_{g} / R\right)^{2}$, which yielded the first few local terms known in the standard string theory, whose coupling constants were completely determined through the bilocal correlator. In Ref. [6], perturbative corrections to the obtained string effective action arising due to perturbative gluons propagating in the nonperturbative QCD vacuum have been calculated, and in Ref. [7] a possible way of the solution of the problem of crumpling of the string world-sheet has been proposed.

However, it still remains unclear how one can get a mechanism of integration over string worldsheets in QCD, since up to now the construction of the string effective action has been performed on the surface of the minimal area (cf. Eq. (4)). Elaboration out of such a mechanism would eventually completely solve the task of finding a string representation of QCD.

The present paper is devoted to be a first step in this direction. To this end, we shall simplify the problem under study by considering the related Dual Abelian Higgs Model (DAHM). This model approach is based on the commonly accepted observation that on the phenomenological level, quark confinement in QCD can be explained in terms of the dual Meissner effect $[10,11]$. According to the 't Hooft-Mandelstam picture of confinement, the properties of the QCD string should be similar to the ones of the electric vortex, which emerges between two electrically charged particles immersed into the superconducting medium filled with a condensate of Cooper pairs of magnetic monopoles. In the case of the usual Abelian Higgs Model (AHM), which is a relativistic version of the Ginzburg-Landau theory of superconductivity, such vortices are referred to as Abrikosov-Nielsen-Olesen strings [12].

Thus, it looks reasonable to consider DAHM as a natural laboratory for probing various approaches to the problem of the string representation of QCD (for related investigations of AHM see [11,13-18]). In this respect, in Ref. [14] the so-called path integral duality transformation proposed in Ref. [13] has been applied to the lattice version of AHM in the London limit in order to reformulate the partition function of this theory in terms of the string world-sheet coordinates. Then the same reformulation has been performed in the continuum limit in Ref. [17].

In this paper, we would like to demonstrate the usefulness of the path integral duality transformation for the derivation of the string representation for the generating functionals of gauge field strength tensor and monopole current correlators in DAHM. In particular, we find it useful to study a suitably extended version of this model, which includes gauge fields generated by external electrically charged particles called "quarks". 
The outline of the paper is as follows. In Section 2, we shall derive the string representation for the partition function of such an extended version of DAHM in the London limit. The resulting action will consist of two parts, the first of which will describe the short-range Yukawa interaction between a quark and an antiquark, whereas the second one, corresponding to the long-range confining potential, will contain the integral over all the surfaces encircled by the contour of the quark-antiquark pair.

In Section 3, we generalize the result of Section 2 by introducing external sources of the field strength tensors in order to derive the string representation for the generating functional of gauge field correlators in the London limit of extended DAHM. In particular, we shall calculate the bilocal correlator and demonstrate that its long- and short distance asymptotic behaviours are in agreement with those found in QCD.

In Section 4, we derive the string representation for the generating functional of monopole current correlators and calculate the correlator of two such currents.

Finally, some technical details concerning the path integral duality transformation and the integration over the related Kalb-Ramond field are outlined in two Appendices.

\section{String Representation for the Partition Function of the Extended DAHM in the London Limit}

We shall start with the following expression for the partition function of the extended DAHM

$$
\mathcal{Z}=\int|\Phi| \mathcal{D}|\Phi| \mathcal{D} B_{\mu} \mathcal{D} \theta \exp \left\{-\int d^{4} x\left[\frac{1}{4}\left(F_{\mu \nu}-F_{\mu \nu}^{E}\right)^{2}+\frac{1}{2}\left|D_{\mu} \Phi\right|^{2}+\lambda\left(|\Phi|^{2}-\eta^{2}\right)^{2}\right]\right\}
$$

where $\Phi(x)=|\Phi(x)| \mathrm{e}^{i \theta(x)}$ is an effective Higgs field of "Cooper pairs" of magnetic monopoles, $B_{\mu}$ and $F_{\mu \nu}=\partial_{\mu} B_{\nu}-\partial_{\nu} B_{\mu}$ are the dual gauge field and its field strength tensor, $D_{\mu}=\partial_{\mu}-2 i g B_{\mu}$ is the covariant derivative with $g$ standing for the magnetic coupling constant. Notice that in Eq. (6), $F_{\mu \nu}^{E}$ stands for the field strength tensor generated by external "quarks", defined according to the equation

$$
\partial_{\nu} \tilde{F}_{\mu \nu}^{E} \equiv \frac{1}{2} \varepsilon_{\mu \nu \lambda \rho} \partial_{\nu} F_{\lambda \rho}^{E}=4 \pi j_{\mu}^{E}
$$

with

$$
j_{\mu}^{E}(x) \equiv e \int_{0}^{1} d \tau \frac{d x_{\mu}(\tau)}{d \tau} \delta(x-x(\tau))
$$

standing for the conserved electric current of a quark, which moves along the closed contour $C$, parametrized by the function $x_{\mu}(\tau), 0 \leq \tau \leq 1, x_{\mu}(0)=x_{\mu}(1)$. The electric coupling constant $e$ is related to the magnetic one via Dirac's quantization condition $e g=\frac{n}{2}$, where $n$ is an integer f In what follows, we shall for concreteness restrict ourselves to the case of monopoles possessing the minimal charge, i.e. put $n=1$.

The solution of Eq. (7) reads

$$
F_{\mu \nu}^{E}=4 \pi e \tilde{\Sigma}_{\mu \nu},
$$

\footnotetext{
${ }^{2}$ Here, we have adopted the notations of Ref. [11].
} 
where $\Sigma_{\mu \nu}(x) \equiv \int_{\Sigma} d \sigma_{\mu \nu}(x(\xi)) \delta(x-x(\xi))$ is the so-called vorticity tensor current [16] defined on the string world-sheet $\Sigma$. This world-sheet is parametrized by the four-vector $x_{\mu}(\xi)$, where $\xi=\left(\xi^{1}, \xi^{2}\right)$ is a two-dimensional coordinate. Due to the Stokes theorem, the vorticity tensor current is related to the quark current according to the equation

$$
e \partial_{\nu} \Sigma_{\mu \nu}=j_{\mu}^{E}
$$

In particular, this equation means, that in the case, when there are no external quarks, the vorticity tensor current is conserved, i.e. due to the conservation of electric flux all the strings in this case are closed. Notice, that when external quarks are introduced into the system, some amount of closed strings might survive. From now on, we shall restrict ourselves to the sector of the theory with open strings ending at quarks and antiquarks only.

In the London limit, $\lambda \rightarrow \infty$, the radial part of the monopole field becomes fixed to its v.e.v., $|\Phi| \rightarrow \eta$, and the partition function (6) takes the form

$$
\mathcal{Z}=\int \mathcal{D} B_{\mu} \mathcal{D} \theta^{\text {sing. }} \mathcal{D} \theta^{\text {reg. }} \exp \left\{-\int d^{4} x\left[\frac{1}{4}\left(F_{\mu \nu}-F_{\mu \nu}^{E}\right)^{2}+\frac{\eta^{2}}{2}\left(\partial_{\mu} \theta-2 g B_{\mu}\right)^{2}\right]\right\},
$$

where from now on constant normalization factors will be omitted. In Eq. (8), we have performed a decomposition of the phase of the magnetic Higgs field $\theta=\theta^{\text {sing. }}+\theta^{\text {reg. }}$, where $\theta^{\text {sing. }}(x)$ obeys the equation (see e.g. [13])

$$
\varepsilon_{\mu \nu \lambda \rho} \partial_{\lambda} \partial_{\rho} \theta^{\text {sing. }}(x)=2 \pi \Sigma_{\mu \nu}(x)
$$

and describes a given electric string configuration, whereas $\theta^{\text {reg. }}(x)$ stands for a single-valued fluctuation around this configuration. Notice also, that as it has been shown in Ref. [17], the integration measure over the field $\theta$ factorizes into the product of measures over the fields $\theta^{\text {sing. }}$ and $\theta^{\text {reg. }}$.

Performing the path integral duality transformation of Eq. (8) along the lines described in Ref. [13], we get

$$
\begin{aligned}
\mathcal{Z}=\exp \left(-(2 \pi e)^{2} \int d^{4} x \Sigma_{\mu \nu}^{2}\right) & \int \mathcal{D} B_{\mu} \int \mathcal{D} h_{\mu \nu} \int \mathcal{D} x_{\mu}(\xi) \exp \left\{\int d ^ { 4 } x \left[-\frac{1}{12 \eta^{2}} H_{\mu \nu \lambda}^{2}+i \pi h_{\mu \nu} \Sigma_{\mu \nu}-\right.\right. \\
& \left.\left.-\frac{1}{4} F_{\mu \nu}^{2}-i \tilde{F}_{\mu \nu}\left(g h_{\mu \nu}+2 \pi i e \Sigma_{\mu \nu}\right)\right]\right\}
\end{aligned}
$$

where $H_{\mu \nu \lambda} \equiv \partial_{\mu} h_{\nu \lambda}+\partial_{\lambda} h_{\mu \nu}+\partial_{\nu} h_{\lambda \mu}$ is the field strength tensor of an antisymmetric tensor field $h_{\mu \nu}$ (the so-called Kalb-Ramond field). Next, by carrying out the integration over the field $B_{\mu}$ in Eq. (10), we obtain

$$
\mathcal{Z}=\int \mathcal{D} x_{\mu}(\xi) \int \mathcal{D} h_{\mu \nu} \exp \left\{\int d^{4} x\left[-\frac{1}{12 \eta^{2}} H_{\mu \nu \lambda}^{2}-\frac{1}{4 e^{2}} h_{\mu \nu}^{2}-i \pi h_{\mu \nu} \Sigma_{\mu \nu}\right]\right\} .
$$

The details of the derivation of Eqs. (10) and (11) are outlined in the Appendix 1.

Finally, the Gaussian integration over the field $h_{\mu \nu}$ in Eq. (11) (see Appendix 2) leads to the following expression for the partition function (8) 


$$
\mathcal{Z}=\int \mathcal{D} x_{\mu}(\xi) \exp \left\{-\pi^{2} \int_{\Sigma} d \sigma_{\lambda \nu}(x) \int_{\Sigma} d \sigma_{\mu \rho}(y) D_{\lambda \nu, \mu \rho}(x-y)\right\}
$$

In Eq. (12), the propagator of the field $h_{\mu \nu}$ has the following form

$$
D_{\lambda \nu, \mu \rho}(x) \equiv D_{\lambda \nu, \mu \rho}^{(1)}(x)+D_{\lambda \nu, \mu \rho}^{(2)}(x)
$$

where

$$
\begin{gathered}
D_{\lambda \nu, \mu \rho}^{(1)}(x)=\frac{\eta^{3}}{8 \pi^{2} e} \frac{K_{1}}{|x|}\left(\delta_{\lambda \mu} \delta_{\nu \rho}-\delta_{\mu \nu} \delta_{\lambda \rho}\right) \\
D_{\lambda \nu, \mu \rho}^{(2)}(x)=\frac{e \eta}{4 \pi^{2} x^{2}}\left\{\left[\frac{K_{1}}{|x|}+\frac{m}{2}\left(K_{0}+K_{2}\right)\right]\left(\delta_{\lambda \mu} \delta_{\nu \rho}-\delta_{\mu \nu} \delta_{\lambda \rho}\right)+\right. \\
+\frac{1}{2|x|}\left[3\left(\frac{m^{2}}{4}+\frac{1}{x^{2}}\right) K_{1}+\frac{3 m}{2|x|}\left(K_{0}+K_{2}\right)+\frac{m^{2}}{4} K_{3}\right] . \\
\left.\cdot\left(\delta_{\lambda \rho} x_{\mu} x_{\nu}+\delta_{\mu \nu} x_{\lambda} x_{\rho}-\delta_{\mu \lambda} x_{\nu} x_{\rho}-\delta_{\nu \rho} x_{\mu} x_{\lambda}\right)\right\} .
\end{gathered}
$$

Here $K_{i} \equiv K_{i}(m|x|), i=0,1,2,3$, stand for the modified Bessel functions, and $m \equiv \frac{\eta}{e}$ is the mass of the dual gauge boson generated by the Higgs mechanism. Due to the Stokes theorem, the term

$$
\int_{\Sigma} d \sigma_{\lambda \nu}(x) \int_{\Sigma} d \sigma_{\mu \rho}(y) D_{\lambda \nu, \mu \rho}^{(2)}(x-y)
$$

can be rewritten as a boundary one (see Appendix 2), which finally leads to the following representation for the partition function of extended DAHM in the London limit 3

$$
\begin{gathered}
\mathcal{Z}=\exp \left[-\frac{e \eta}{2} \oint_{C} d x_{\mu} \oint_{C} d y_{\mu} \frac{K_{1}(m|x-y|)}{|x-y|}\right] . \\
\cdot \int \mathcal{D} x_{\mu}(\xi) \exp \left[-\frac{\eta^{3}}{4 e} \int_{\Sigma} d \sigma_{\mu \nu}(x) \int_{\Sigma} d \sigma_{\mu \nu}(y) \frac{K_{1}(m|x-y|)}{|x-y|}\right] .
\end{gathered}
$$

The first exponent on the R.H.S. of Eq. (15) leads to the short-range Yukawa potential,

$$
V_{\text {Yuk. }}(R) \propto \frac{1}{R} \mathrm{e}^{-m R}
$$

Notice that since quarks and antiquarks were from the very beginning considered as classical particles, the external contour $C$ explicitly enters the final result. Would one consider them on the quantum level, Eq. (15) must be supplied by a certain prescription of the summation over the contours $[1,11]$.

\footnotetext{
${ }^{3}$ It is worth mentioning, that an analogous expression for the partition function has been obtained and investigated in Ref. [11]. This has been done by making use of techniques different from the ones applied in the present paper.
} 
The integral over string world-sheets on the R.H.S. of Eq. (15) is the essence of the string representation of the partition function. Being carried out in the saddle-point approximation, it results in the last exponent on the R.H.S. of Eq. (15), where the integrals are taken over the surface of the minimal area, encircled by the contour $C, \Sigma_{\text {min. }}$ (cf. Eq. (4)). This term then yields a rising confining quark-antiquark potential (2).

As it has already been discussed in the Introduction, the string tension $\sigma$ in Eq. (2) is nothing else, but the coupling constant of the Nambu-Goto term,

$$
S_{\mathrm{NG}}=\sigma \int d^{2} \xi \sqrt{\hat{g}}
$$

where $\hat{g}$ stands for the determinant of the induced metric tensor $\hat{g}_{a b}(\xi)=\left(\partial_{a} x_{\mu}(\xi)\right)\left(\partial_{b} x_{\mu}(\xi)\right)$ of the string world-sheet, $a, b=1,2$. This term is the first local term in the derivative expansion of the full nonlocal string effective action

$$
S_{\text {eff. }}=\frac{\eta^{3}}{4 e} \int_{\Sigma_{\min }} d \sigma_{\mu \nu}(x) \int_{\Sigma_{\min } .} d \sigma_{\mu \nu}(y) \frac{K_{1}(m|x-y|)}{|x-y|} .
$$

The second local term in this expansion is the so-called rigidity term $[19,20]$

$$
S_{\text {rigidity }}=\frac{1}{\alpha_{0}} \int d^{2} \xi \sqrt{\hat{g}} \hat{g}^{a b}\left(\partial_{a} t_{\mu \nu}(\xi)\right)\left(\partial_{b} t_{\mu \nu}(\xi)\right),
$$

where $t_{\mu \nu}(\xi)=\frac{1}{\sqrt{\hat{g}}} \varepsilon^{a b}\left(\partial_{a} x_{\mu}(\xi)\right)\left(\partial_{b} x_{\nu}(\xi)\right)$ is the extrinsic curvature tensor of the string world-sheet, and $\frac{1}{\alpha_{0}}$ is the inverse bare coupling constant.

Making use of the results of Ref. [5], it is possible to derive from the nonlocal string effective

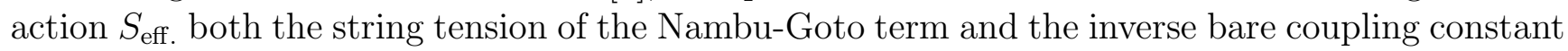
of the rigidity term. The latter one occurs to be finite and reads

$$
\frac{1}{\alpha_{0}}=-\frac{\pi e^{2}}{8}
$$

In particular, one can see that $\frac{1}{\alpha_{0}}<0$, which reflects the stability of strings [20,15,21] 1 . As far as the string tension is concerned, one obtains

$$
\sigma=\pi \eta^{2} K_{0}(a) \simeq \pi \eta^{2} \ln \frac{2}{\gamma a}
$$

where $\gamma=1.781 \ldots$ is the Euler's constant, and $a$ stands for a characteristic small dimensionless parameter. In the London limit, we get $a \sim \frac{m}{M}$, where $M=2 \sqrt{2 \lambda} \eta$ is the monopole mass following from Eq. (6). This mass plays the role of the UV momentum cutoff somehow analogous to the inverse lattice spacing in QCD. Notice, that the logarithmic divergency of the string tension in the Ginzburg-Landau model and AHM is a well known result, which can be obtained directly from the definition of this quantity as a free energy per unit length of the string (see e.g. [22]). Clearly, both the string tension and the inverse bare coupling constant of the rigidity term are not proportional to $g$, which means that these quantities are essentially nonperturbative similarly to the QCD case (cf. Eq. (3)).

\footnotetext{
${ }^{4}$ Notice, that the negative sign of this coupling constant alone does not guarantee stability of strings. In the non-Abelian case, one should elaborate out another mechanisms to ensure this stability and get rid of crumpling of the string world-sheet $[19,7]$.
} 


\section{String Representation for the Generating Functional of Field Strength Correlators}

In this Section, we shall derive the string representation for the generating functional of field strength correlators in the London limit of extended DAHM. From this we shall then obtain an expression for the bilocal correlator and compare it with the one in QCD. Our starting expression for the generating functional reads as follows

$$
\mathcal{Z}\left[S_{\alpha \beta}\right]=\int \mathcal{D} B_{\mu} \mathcal{D} \theta^{\text {sing. }} \mathcal{D} \theta^{\text {reg. }} \exp \left\{-\int d^{4} x\left[\frac{1}{4}\left(F_{\mu \nu}-F_{\mu \nu}^{E}\right)^{2}+\frac{\eta^{2}}{2}\left(\partial_{\mu} \theta-2 g B_{\mu}\right)^{2}+i S_{\mu \nu} \tilde{F}_{\mu \nu}\right]\right\}
$$

where $S_{\mu \nu}$ is a source of the field strength tensor $\tilde{F}_{\mu \nu}=\frac{1}{2} \varepsilon_{\mu \nu \lambda \rho} F_{\lambda \rho}$, which obviously corresponds to the field strength of the usual gauge field $A_{\mu}$ in the AHM. Performing the same transformations which led from Eq. (8) to Eq. (12), we obtain from Eq. (18)

$$
\begin{gathered}
\mathcal{Z}\left[S_{\alpha \beta}\right]=\exp \left[-\int d^{4} x\left(S_{\mu \nu}^{2}+4 \pi i e S_{\mu \nu} \Sigma_{\mu \nu}\right)\right] . \\
\cdot \int \mathcal{D} x_{\mu}(\xi) \exp \left\{-\int d^{4} x d^{4} y\left(\pi \Sigma_{\lambda \nu}(x)-\frac{i}{e} S_{\lambda \nu}(x)\right) D_{\lambda \nu, \mu \rho}(x-y)\left(\pi \Sigma_{\mu \rho}(y)-\frac{i}{e} S_{\mu \rho}(y)\right)\right\} .
\end{gathered}
$$

Let us now derive from the general form (19) of the generating functional the expression for the bilocal correlator of the field strength tensors. The result reads

$$
\begin{gathered}
\left\langle\tilde{F}_{\lambda \nu}(x) \tilde{F}_{\mu \rho}(y)\right\rangle=\left.\frac{1}{\mathcal{Z}[0]} \frac{\delta^{2} \mathcal{Z}\left[S_{\alpha \beta}\right]}{\delta S_{\lambda \nu}(x) \delta S_{\mu \rho}(y)}\right|_{S_{\alpha \beta}=0}= \\
=\left(\delta_{\lambda \mu} \delta_{\nu \rho}-\delta_{\lambda \rho} \delta_{\mu \nu}\right) \delta(x-y)+\frac{2}{e^{2}} D_{\lambda \nu, \mu \rho}(x-y)- \\
-4 \pi^{2}\left\langle\left(2 e \Sigma_{\lambda \nu}(x)-\frac{1}{e} \int_{\Sigma} d \sigma_{\alpha \beta}(z) D_{\alpha \beta, \lambda \nu}(z-x)\right)\left(2 e \Sigma_{\mu \rho}(x)-\frac{1}{e} \int_{\Sigma} d \sigma_{\gamma \zeta}(u) D_{\gamma \zeta, \mu \rho}(u-y)\right)\right\rangle_{x_{\mu}(\xi)}
\end{gathered}
$$

where

$$
\langle\ldots\rangle_{x_{\mu}(\xi)} \equiv \frac{\int \mathcal{D} x_{\mu}(\xi)(\ldots) \exp \left[-\pi^{2} \int_{\Sigma} d \sigma_{\alpha \beta}(z) \int_{\Sigma} d \sigma_{\gamma \zeta}(u) D_{\alpha \beta, \gamma \zeta}(z-u)\right]}{\int \mathcal{D} x_{\mu}(\xi) \exp \left[-\pi^{2} \int_{\Sigma} d \sigma_{\alpha \beta}(z) \int_{\Sigma} d \sigma_{\gamma \zeta}(u) D_{\alpha \beta, \gamma \zeta}(z-u)\right]}
$$

is the average over the string world-sheets, and the term with the $\delta$-function on the R.H.S. of Eq. (20) corresponds to the free contribution to the correlator.

Let us next study the large distance asymptotics of Eqs. (13) and (14), i.e. consider these equations at $|x| \gg \frac{1}{m}$. Then one can see, that due to the large distance asymptotics of the modified Bessel functions, the propagator $D_{\lambda \nu, \mu \rho}(x)$ has the order of magnitude $\frac{\eta^{4}}{e^{2}}$. Therefore, at 
such distances the absolute value of the second term on the R.H.S. of Eq. (20) is much larger than the absolute value of the last term, provided that the following inequality holds

$$
\frac{\eta^{2}|\Sigma|}{e} \ll 1
$$

where $|\Sigma|$ stands for the area of the surface $\Sigma$. In what follows, we shall restrict ourselves to the case of small enough $\eta$ and $g$, for which this inequality is valid, and consequently the last term on the R.H.S. of Eq. (20) can be disregarded w.r.t. the second one.

Following the SVM $[2,8]$, let us parametrize the bilocal correlator of the field strength tensors by the two Lorentz structures similarly to Eq. (5)

$$
\begin{gathered}
\left\langle\tilde{F}_{\lambda \nu}(x) \tilde{F}_{\mu \rho}(0)\right\rangle=\left(\delta_{\lambda \mu} \delta_{\nu \rho}-\delta_{\lambda \rho} \delta_{\nu \mu}\right) D\left(x^{2}\right)+ \\
+\frac{1}{2}\left[\partial_{\lambda}\left(x_{\mu} \delta_{\nu \rho}-x_{\rho} \delta_{\nu \mu}\right)+\partial_{\nu}\left(x_{\rho} \delta_{\lambda \mu}-x_{\mu} \delta_{\lambda \rho}\right)\right] D_{1}\left(x^{2}\right) .
\end{gathered}
$$

Then in the approximation (21), by virtue of Eqs. (13) and (14), we arrive at the following expressions for the functions $D$ and $D_{1}$

$$
D\left(x^{2}\right)=\frac{m^{3}}{4 \pi^{2}} \frac{K_{1}}{|x|},
$$

and

$$
D_{1}\left(x^{2}\right)=\frac{m}{2 \pi^{2} x^{2}}\left[\frac{K_{1}}{|x|}+\frac{m}{2}\left(K_{0}+K_{2}\right)\right] .
$$

In Eq. (23), we have discarded the free $\delta$-function type contribution, since we are working at large distances so that $x \neq 0$. The asymptotic behaviour of the coefficient functions (23) and (24) in the limit $|x| \gg \frac{1}{m}$ under study is then given by

$$
D \longrightarrow \frac{m^{4}}{4 \sqrt{2} \pi^{\frac{3}{2}}} \frac{\mathrm{e}^{-m|x|}}{(m|x|)^{\frac{3}{2}}},
$$

and

$$
D_{1} \longrightarrow \frac{m^{4}}{2 \sqrt{2} \pi^{\frac{3}{2}}} \frac{\mathrm{e}^{-m|x|}}{(m|x|)^{\frac{5}{2}}} .
$$

For bookkeeping purposes, let us also list here the asymptotic behaviour of the functions (23) and (24) in the opposite case, $|x| \ll \frac{1}{m}$. It reads

$$
D \longrightarrow \frac{m^{2}}{4 \pi^{2} x^{2}},
$$

and

$$
D_{1} \longrightarrow \frac{1}{\pi^{2}|x|^{4}}
$$


One can now see that according to the lattice data [9] the asymptotic behaviours (25) and (26) are very similar to the large distance ones of the nonperturbative parts of the functions $D^{\mathrm{QCD}}$ and $D_{1}^{\mathrm{QCD}}$, which parametrize the gauge-invariant bilocal correlator of gluonic field strength tensors in QCD (see Eq. (5)). In particular, both functions decrease exponentially, and the function $D$ is much larger then the function $D_{1}$ due to the preexponential power-like behaviour. We also see that the dual gauge boson mass $m$ corresponds to the inverse correlation length of the vacuum $T_{g}^{-1}$. In particular, in the string limit of QCD, studied in Refs. [5-7], when $T_{g} \rightarrow 0$ while the value of the string tension is kept fixed, $m$ corresponds to $\sqrt{\frac{D^{\mathrm{QCD}}(0)}{\sigma}}$.

Moreover, the short distance asymptotic behaviours (27) and (28) are also in line with the results obtained within the SVM of QCD in the lowest order of perturbation theory. Namely, at such distances the function $D_{1}^{\mathrm{QCD}}$ to the lowest order also behaves as $\frac{1}{|x|^{4}}$ (which is just due to the one-gluon-exchange contribution) and is much larger than the function $D^{\mathrm{QCD}}$ to the same order. Let us however stress once more, that Eqs. (27) and (28) contain only a part of the full information about the asymptotic behaviours of the functions $D$ and $D_{1}$ at small distances. The remaining information is contained in the omitted last term on the R.H.S. of Eq. (20), which at small distances might become important and modify the asymptotic behaviours (27) and (28).

The above mentioned similarity in the large- and short distance asymptotic behaviours of the functions $D$ and $D_{1}$, which parametrize the bilocal correlator of the field strength tensors in DAHM and the gauge-invariant correlator in QCD, thus supports the original conjecture by 't Hooft and Mandelstam concerning the dual Meissner nature of confinement.

\section{String Representation for the Generating Functional of the Monopole Current Correlators}

In this Section, we shall present the string representation for the generating functional of the monopole current correlators in the London limit of extended DAHM. Such a representation can be derived by virtue of the same path integral duality transformation studied above. After that, we shall get from the obtained generating functional the correlator of two monopole currents and by making use of it rederive via the equations of motion the coefficient function $D$ in the bilocal correlator of the field strength tensors.

In the London limit, the generating functional of the monopole currents reads

$$
\hat{\mathcal{Z}}\left[J_{\mu}\right]=\int \mathcal{D} B_{\mu} \mathcal{D} \theta^{\text {sing. }} \mathcal{D} \theta^{\text {reg. }} \exp \left\{\int d^{4} x\left[-\frac{1}{4}\left(F_{\mu \nu}-F_{\mu \nu}^{E}\right)^{2}-\frac{\eta^{2}}{2}\left(\partial_{\mu} \theta-2 g B_{\mu}\right)^{2}+J_{\mu} j_{\mu}\right]\right\},
$$

where $j_{\mu} \equiv-2 g \eta^{2}\left(\partial_{\mu} \theta-2 g B_{\mu}\right)$ is just the magnetic monopole current .

Performing the duality transformation, we get the following string representation for $\hat{\mathcal{Z}}\left[J_{\mu}\right]$

$$
\begin{aligned}
\hat{\mathcal{Z}}\left[J_{\mu}\right]= & \exp \left[\frac{m^{2}}{2} \int d^{4} x J_{\mu}^{2}(x)\right] \int \mathcal{D} x_{\mu}(\xi) \exp \left[-\pi^{2} \int_{\Sigma} d \sigma_{\alpha \beta}(z) \int_{\Sigma} d \sigma_{\gamma \zeta}(u) D_{\alpha \beta, \gamma \zeta}(z-u)\right] . \\
& \cdot \exp \left\{2 g \varepsilon _ { \lambda \nu \alpha \beta } \int d ^ { 4 } x d ^ { 4 } y \left[-\frac{g}{2} \varepsilon_{\mu \rho \gamma \delta}\left(\frac{\partial^{2}}{\partial x_{\alpha} \partial y_{\gamma}} D_{\lambda \nu, \mu \rho}(x-y)\right) J_{\beta}(x) J_{\delta}(y)+\right.\right.
\end{aligned}
$$

\footnotetext{
${ }^{5}$ Rigorously speaking, this is a current of the monopole Cooper pairs.
} 


$$
\left.\left.+\pi \Sigma_{\mu \rho}(y)\left(\frac{\partial}{\partial x_{\alpha}} D_{\lambda \nu, \mu \rho}(x-y)\right) J_{\beta}(x)\right]\right\} .
$$

Varying now Eq. (29) twice w.r.t. $J_{\mu}$, setting then $J_{\mu}$ equal to zero, and dividing the result by $\hat{\mathcal{Z}}[0]$, we arrive at the following expression for the correlator of two monopole currents

$$
\begin{gathered}
\left\langle j_{\beta}(x) j_{\sigma}(y)\right\rangle=m^{2} \delta_{\beta \sigma} \delta(x-y)+4 g^{2} \varepsilon_{\lambda \nu \alpha \beta} \varepsilon_{\mu \rho \gamma \sigma}\left[-\frac{1}{2} \frac{\partial^{2}}{\partial x_{\alpha} \partial y_{\gamma}} D_{\lambda \nu, \mu \rho}(x-y)+\right. \\
\left.+\pi^{2}\left\langle\int_{\Sigma} d \sigma_{\delta \zeta}(z) \int_{\Sigma} d \sigma_{\chi \varphi}(u)\left(\frac{\partial}{\partial x_{\alpha}} D_{\lambda \nu, \delta \zeta}(x-z)\right)\left(\frac{\partial}{\partial y_{\gamma}} D_{\mu \rho, \chi \varphi}(y-u)\right)\right\rangle_{x_{\mu}(\xi)}\right] .
\end{gathered}
$$

It is straightforward to see that the contribution of the term (14) to the R.H.S. of Eq. (30) vanishes, whereas the contribution of Eq. (13) to the second term in the square brackets on the R.H.S. of Eq. (30) can be disregarded w.r.t. its contribution to the first term, provided that the inequality (21) holds. Within this approximation, making use of the equation [2]

$$
\left\langle j_{\beta}(x) j_{\sigma}(y)\right\rangle=\left(\frac{\partial^{2}}{\partial x_{\mu} \partial y_{\mu}} \delta_{\beta \sigma}-\frac{\partial^{2}}{\partial x_{\beta} \partial y_{\sigma}}\right) D\left((x-y)^{2}\right),
$$

which follows from Eq. (22) due to equations of motion, we recover from Eqs. (30), (31), and (13) the expression for the function $D$ given by Eq. (23).

Notice in conclusion, that only the function $D$ can be obtained from the correlator (30) due to the independence of the latter of the function $D_{1}$.

\section{Summary and Outlook}

In the present paper, we have derived the string representation for the partition function of DAHM extended by introducing gauge fields of external currents of electrically charged particles ("quarks"), in the London limit. Such a representation yielded the confining and the Yukawa parts of the quark-antiquark interaction potential. By the local expansion of the obtained nonlocal string effective action, one gets, in particular, the corresponding expressions for the string tension of the Nambu-Goto term and the inverse bare coupling constant of the rigidity term. Those occured to be positive and negative respectively, which confirms the stability of strings (cf. also $[20,15,21]$ ). Besides that, both of these quantities are not proportional to the coupling constant, which means that the string nature of DAHM is of the same nonperturbative kind as the one of QCD.

In Section 3, we have obtained the string representation for the generating functional of the field strength correlators. In a certain approximation (see Eq. (21)), this generating functional determined then the expressions of the two coefficient functions, $D$ and $D_{1}$ (cf. Eqs. (23) and (24)), which parametrize the bilocal correlator. Those occured to be quite similar to the corresponding functions in QCD. In particular, it turned out that the large distance asymptotic behaviour of the obtained functions in the extended DAHM was in agreement with the existing lattice data [9] concerning the corresponding behaviours of the nonperturbative parts of these functions in QCD. We have also argued that the mass of the dual gauge boson in our approach corresponds to the inverse correlation length of the vacuum in QCD. These results together with SVM support the 't Hooft-Mandelstam conjecture about the dual Meissner nature of confinement. 
Finally, in Section 4, we have obtained the string representation for the generating functional of monopole current correlators in the extended DAHM. Then, by making use of the equations of motion, and the string representation for the correlator of two monopole currents, we have rederived the coefficient function $D$, which confirms the correctness of both approaches.

Thus, we have demonstrated the relevance of the extended DAHM for the description of the string properties of confinement in QCD according to SVM and the 't Hooft-Mandelstam scenario. And vice versa, our results also support the validity of the bilocal approximation in SVM of QCD and provide us with some new insights concerning the structure of the QCD vacuum. In conclusion, all this shows the usefulness of the string representation of extended DAHM obtained on the basis of the path integral duality transformation.

Clearly, it is now a challenge to apply this approach to the realistic non-Abelian case of QCD. In the spirit of this paper, it is in particular interesting to study dual QCD in the framework of the so-called Abelian projection method $[23,18]$. Work in this direction is now in progress.

\section{Acknowledgments}

We are indebted to A. Di Giacomo, H. Dorn, H. Kleinert, M.I. Polikarpov, Chr. Preitschopf, and Yu.A. Simonov for useful discussions. One of us (D.A.) would also like to thank the theory group of the Quantum Field Theory Department of the Institute of Physics of the Humboldt University of Berlin for kind hospitality and Graduiertenkolleg Elementarteilchenphysik for financial support.

\section{Appendix 1. Derivation of Eqs. (10) and (11).}

In this Appendix, we shall outline some details of the derivation of Eqs. (10) and (11). Firstly, one can linearize the term $\frac{\eta^{2}}{2}\left(\partial_{\mu} \theta-2 g B_{\mu}\right)^{2}$ in the exponent on the R.H.S. of Eq. (8) and carry out the integral over $\theta^{\text {reg. }}$ as follows

$$
\begin{gathered}
\int \mathcal{D} \theta^{\text {reg. }} \exp \left\{-\frac{\eta^{2}}{2} \int d^{4} x\left(\partial_{\mu} \theta-2 g B_{\mu}\right)^{2}\right\}= \\
=\int \mathcal{D} C_{\mu} \mathcal{D} \theta^{\text {reg. }} \exp \left\{\int d^{4} x\left[-\frac{1}{2 \eta^{2}} C_{\mu}^{2}+i C_{\mu}\left(\partial_{\mu} \theta-2 g B_{\mu}\right)\right]\right\}= \\
=\int \mathcal{D} C_{\mu} \delta\left(\partial_{\mu} C_{\mu}\right) \exp \left\{\int d^{4} x\left[-\frac{1}{2 \eta^{2}} C_{\mu}^{2}+i C_{\mu}\left(\partial_{\mu} \theta^{\text {sing. }}-2 g B_{\mu}\right)\right]\right\} .
\end{gathered}
$$

The constraint $\partial_{\mu} C_{\mu}=0$ can be uniquely resolved by representing $C_{\mu}$ in the form $C_{\mu}=$ $\frac{1}{2} \varepsilon_{\mu \nu \lambda \rho} \partial_{\nu} h_{\lambda \rho}$, where $h_{\lambda \rho}$ stands for an antisymmetric tensor field. Notice, that the number of degrees of freedom during such a replacement is conserved, since both of the fields $C_{\mu}$ and $h_{\mu \nu}$ have three independent components.

Then, taking into account the relation (9) between $\theta^{\text {sing. }}$ and $\Sigma_{\mu \nu}$, we get from Eq. (A1.1)

$$
\begin{gathered}
\int \mathcal{D} \theta^{\text {sing. }} \mathcal{D} \theta^{\text {reg. }} \exp \left\{-\frac{\eta^{2}}{2} \int d^{4} x\left(\partial_{\mu} \theta-2 g B_{\mu}\right)^{2}\right\}= \\
=\int \mathcal{D} h_{\mu \nu} \mathcal{D} x_{\mu}(\xi) \exp \left\{\int d^{4} x\left[-\frac{1}{12 \eta^{2}} H_{\mu \nu \lambda}^{2}+i \pi h_{\mu \nu} \Sigma_{\mu \nu}-i g \varepsilon_{\mu \nu \lambda \rho} B_{\mu} \partial_{\nu} h_{\lambda \rho}\right]\right\} .
\end{gathered}
$$


In the derivation of Eq. (A1.2), we have replaced $\mathcal{D} \theta^{\text {sing. }}$ by $\mathcal{D} x_{\mu}(\xi)$ (since the surface $\Sigma$, parametrized by $x_{\mu}(\xi)$, is just the surface, at which the field $\theta$ is singular) and, for simplicity, have discarded the Jacobian arising during such a change of the integration variable 0 .

Bringing now together Eqs. (8) and (A1.2), we arrive at Eq. (10). In the literature, the above described sequence of transformations of integration variables is usually called "path integral duality transformation". In particular, it has been applied in Ref. [13] to the model with a global $U(1)$-symmetry.

Let us now derive Eq. (11). To this end, we find it convenient to rewrite

$$
\exp \left(-\frac{1}{4} \int d^{4} x F_{\mu \nu}^{2}\right)=\int \mathcal{D} G_{\mu \nu} \exp \left\{\int d^{4} x\left[-G_{\mu \nu}^{2}+i \tilde{F}_{\mu \nu} G_{\mu \nu}\right]\right\}
$$

after which the $B_{\mu}$-integration in Eq. (10) yields

$$
\begin{gathered}
\int \mathcal{D} B_{\mu} \exp \left\{-\int d^{4} x\left[\frac{1}{4} F_{\mu \nu}^{2}+i \tilde{F}_{\mu \nu}\left(g h_{\mu \nu}+2 \pi i e \Sigma_{\mu \nu}\right)\right]\right\}= \\
=\int \mathcal{D} G_{\mu \nu} \exp \left(-\int d^{4} x G_{\mu \nu}^{2}\right) \delta\left(\varepsilon_{\mu \nu \lambda \rho} \partial_{\mu}\left(G_{\lambda \rho}-g h_{\lambda \rho}-2 \pi i e \Sigma_{\lambda \rho}\right)\right)= \\
=\int \mathcal{D} \Lambda_{\mu} \exp \left[-\int d^{4} x\left(g h_{\mu \nu}+2 \pi i e \Sigma_{\mu \nu}+\partial_{\mu} \Lambda_{\nu}-\partial_{\nu} \Lambda_{\mu}\right)^{2}\right] .
\end{gathered}
$$

In the last line of Eq. (A1.3), the constraint

$$
\varepsilon_{\mu \nu \lambda \rho} \partial_{\mu}\left(G_{\lambda \rho}-g h_{\lambda \rho}-2 \pi i e \Sigma_{\lambda \rho}\right)=0
$$

has been resolved by setting $G_{\lambda \rho}=g h_{\lambda \rho}+2 \pi i e \Sigma_{\lambda \rho}+\partial_{\lambda} \Lambda_{\rho}-\partial_{\rho} \Lambda_{\lambda}$.

Finally, by performing in Eq. (A1.3) the hypergauge transformation $h_{\mu \nu} \rightarrow h_{\mu \nu}+\partial_{\mu} \lambda_{\nu}-\partial_{\nu} \lambda_{\mu}$ and fixing the gauge by choosing $\lambda_{\mu}=-\frac{1}{g} \Lambda_{\mu}$ (see e.g. Ref. [15]), we arrive, omitting the measure factor $\mathcal{D} \Lambda_{\mu}$, at Eq. (11).

\section{Appendix 2. Integration over the Kalb-Ramond Field in Eq. (11).}

Let us carry out the following integration over the Kalb-Ramond field

$$
\mathcal{Z}=\int D h_{\mu \nu} \exp \left[\int d x\left(-\frac{1}{12 \eta^{2}} H_{\mu \nu \lambda}^{2}-\frac{1}{4 e^{2}} h_{\mu \nu}^{2}-i \pi h_{\mu \nu} \Sigma_{\mu \nu}\right)\right] .
$$

To this end, it is necessary to substitute the saddle-point value of the integral (A2.1) back into the integrand. The saddle-point equation in the momentum representation reads

$$
\frac{1}{2 \eta^{2}}\left(p^{2} h_{\nu \lambda}^{\text {extr. }}(p)+p_{\lambda} p_{\mu} h_{\mu \nu}^{\text {extr. }}(p)+p_{\mu} p_{\nu} h_{\lambda \mu}^{\text {extr. }}(p)\right)+\frac{1}{2 e^{2}} h_{\nu \lambda}^{\text {extr. }}(p)=-i \pi \Sigma_{\nu \lambda}(p) .
$$

This equation can be most easily solved by rewriting it in the following way

$$
\left(p^{2} \mathbf{P}_{\lambda \nu, \alpha \beta}+m^{2} \mathbf{1}_{\lambda \nu, \alpha \beta}\right) h_{\alpha \beta}^{\text {extr. }}(p)=-2 \pi i \eta^{2} \Sigma_{\lambda \nu}(p),
$$

where we have introduced the following projection operators

\footnotetext{
${ }^{6}$ For the case when the surface $\Sigma$ has a spherical topology, this Jacobian has been calculated in Ref. [17].
} 


$$
\mathbf{P}_{\mu \nu, \lambda \rho} \equiv \frac{1}{2}\left(\mathcal{P}_{\mu \lambda} \mathcal{P}_{\nu \rho}-\mathcal{P}_{\mu \rho} \mathcal{P}_{\nu \lambda}\right)
$$

and

$$
\mathbf{1}_{\mu \nu, \lambda \rho} \equiv \frac{1}{2}\left(\delta_{\mu \lambda} \delta_{\nu \rho}-\delta_{\mu \rho} \delta_{\nu \lambda}\right)
$$

with $\mathcal{P}_{\mu \nu} \equiv \delta_{\mu \nu}-\frac{p_{\mu} p_{\nu}}{p^{2}}$. These projection operators obey the following relations

$$
\begin{gathered}
\mathbf{1}_{\mu \nu, \lambda \rho}=-\mathbf{1}_{\nu \mu, \lambda \rho}=-\mathbf{1}_{\mu \nu, \rho \lambda}=\mathbf{1}_{\lambda \rho, \mu \nu}, \\
\mathbf{1}_{\mu \nu, \lambda \rho} \mathbf{1}_{\lambda \rho, \alpha \beta}=\mathbf{1}_{\mu \nu, \alpha \beta}
\end{gathered}
$$

(the same relations hold for $\mathbf{P}_{\mu \nu, \lambda \rho}$ ), and

$$
\mathbf{P}_{\mu \nu, \lambda \rho}(\mathbf{1}-\mathbf{P})_{\lambda \rho, \alpha \beta}=0 .
$$

By virtue of properties (A2.3)-(A2.5), the solution of Eq. (A2.2) reads

$$
h_{\lambda \nu}^{\text {extr. }}(p)=-\frac{2 \pi i \eta^{2}}{p^{2}+m^{2}}\left[\mathbf{1}+\frac{p^{2}}{m^{2}}(\mathbf{1}-\mathbf{P})\right]_{\lambda \nu, \alpha \beta} \Sigma_{\alpha \beta}(p),
$$

which, once being substituted back into partition function (A2.1), yields for it the following expression

$$
\mathcal{Z}=\exp \left\{-\pi^{2} \eta^{2} \int \frac{d^{4} p}{(2 \pi)^{4}} \frac{1}{p^{2}+m^{2}}\left[\mathbf{1}+\frac{p^{2}}{m^{2}}(\mathbf{1}-\mathbf{P})\right]_{\mu \nu, \alpha \beta} \Sigma_{\mu \nu}(-p) \Sigma_{\alpha \beta}(p)\right\} .
$$

Rewriting Eq. (A2.6) in the coordinate representation we arrive at Eq. (12).

Let us now prove that the term proportional to the projection operator $\mathbf{1}-\mathbf{P}$ on the R.H.S. of Eq. (A2.6) indeed yields in the coordinate representation the boundary term, i.e. Eq. (14). One has

$$
p^{2}(\mathbf{1}-\mathbf{P})_{\lambda \nu, \alpha \beta}=\frac{1}{2}\left(\delta_{\nu \beta} p_{\lambda} p_{\alpha}+\delta_{\lambda \alpha} p_{\nu} p_{\beta}-\delta_{\nu \alpha} p_{\lambda} p_{\beta}-\delta_{\lambda \beta} p_{\nu} p_{\alpha}\right) .
$$

By making use of Eq. (A2.7), the term

$$
-\pi^{2} \eta^{2} \int \frac{d^{4} p}{(2 \pi)^{4}} \frac{1}{p^{2}+m^{2}} \frac{p^{2}}{m^{2}}(\mathbf{1}-\mathbf{P})_{\mu \nu, \alpha \beta} \int d^{4} x \int d^{4} y \mathrm{e}^{i p(y-x)} \Sigma_{\mu \nu}(x) \Sigma_{\alpha \beta}(y)
$$

under study, after carrying out the integration over $p$, reads

$$
\frac{\eta^{2}}{2 m} \int d^{4} x \Sigma_{\mu \nu}(x) \int d^{4} y \Sigma_{\nu \beta}(y) \frac{\partial^{2}}{\partial x_{\mu} \partial y_{\beta}} \frac{K_{1}(m|x-y|)}{|x-y|} .
$$

Acting in Eq. (A2.8) straightforwardly with the derivatives, we arrive at Eq. (14). However, one can perform the partial integration, which gives the boundary term

$$
-\frac{e^{2} m}{2} \oint_{C} d x_{\mu} \oint_{C} d y_{\mu} \frac{K_{1}(m|x-y|)}{|x-y|}
$$

in which one can recognize the argument of the first exponent standing on the R.H.S. of Eq. (15). 


\section{References}

[1] A.M. Polyakov, Gauge Fields and Strings (Harwood Academic Publishers, Chur, Switzerland, 1987).

[2] Yu.A. Simonov, Phys. Usp. 39 (1996) 313.

[3] K.G. Wilson, Phys. Rev. D 10 (1974) 2445.

[4] I.-J. Ford et al., Phys. Lett. B 208 (1988) 286; E. Laermann et al., Nucl. Phys. B (Proc. Suppl.) 26 (1992) 268.

[5] D.V. Antonov, D. Ebert, and Yu.A. Simonov, Mod. Phys. Lett. A 11 (1996) 1905.

[6] D.V. Antonov and D. Ebert, Mod. Phys. Lett. A 12 (1997) 2047.

[7] D.V. Antonov and D. Ebert, preprints HUB-EP-98-15 and hep-ph/9802353 (1998) (Phys. Rev. $\mathrm{D}$, in press).

[8] H.G. Dosch, Phys. Lett. B 190 (1987) 177; H.G. Dosch and Yu.A. Simonov, Phys. Lett. B 205 (1988) 339; Yu.A. Simonov, Nucl. Phys. B 324 (1989) 67; Sov. J. Nucl. Phys. 54 (1991) 115; H.G. Dosch, Prog. Part. Nucl. Phys. 33 (1994) 121.

[9] A. Di Giacomo and H. Panagopoulos, Phys. Lett. B 285 (1992) 133; M. D'Elia, A. Di Giacomo, and E. Meggiolaro, Phys. Lett. B 408 (1997) 315; A. Di Giacomo, E. Meggiolaro, and H. Panagopoulos, Nucl. Phys. B 483 (1997) 371; Nucl. Phys. Proc. Suppl. A 54 (1997) 343.

[10]S. Mandelstam, Phys. Rep. C 23 (1976) 245; G. 't Hooft, in: High Energy Physics, ed. A. Zichichi (Editrice Compositori, Bologna, 1976).

[11]H. Kleinert, Gauge Fields in Condensed Matter, Vol. 1: Superflow and Vortex Lines. Disorder Fields, Phase Transitions (World Scientific, Singapore, 1989); Phys. Lett. B 246 (1990) 127; ibid. B 293 (1992) 168; M. Kiometzis, H. Kleinert, and A.M.J. Schakel, Fortschr. Phys. 43 (1995) 697; H. Kleinert, preprint cond-mat/9503030 (1995).

[12]A.A. Abrikosov, Sov. Phys. JETP 32 (1957) 1442; H.B. Nielsen and P. Olesen, Nucl. Phys. B 61 (1973) 45.

[13]K. Lee, Phys. Rev. D 48 (1993) 2493.

[14]M.I. Polikarpov, U.-J. Wiese, and M.A. Zubkov, Phys. Lett. B 309 (1993) 133.

[15]P. Orland, Nucl. Phys. B 428 (1994) 221.

[16]M. Sato and S. Yahikozawa, Nucl. Phys. B 436 (1995) 100.

[17]E.T. Akhmedov, M.N. Chernodub, M.I. Polikarpov, and M.A. Zubkov, Phys. Rev. D 53 (1996) 2087.

[18]M.N. Chernodub and M.I. Polikarpov, preprints hep-th/9710205 and ITEP-TH-55-97 (1997).

[19]A.M. Polyakov, Nucl. Phys. B 268 (1986) 406.

[20]H. Kleinert, Phys. Lett. B 174 (1986) 335; ibid. B 211 (1988) 151.

[21]M.C. Diamantini, F. Quevedo, and C.A. Trugenberger, Phys. Lett. B 396 (1997) 115; M.C.

Diamantini and C.A. Trugenberger, Phys. Lett. B 421 (1998) 196; preprint hep-th/9803046 (1998).

[22]E.M. Lifshitz and L.P. Pitaevski, Statistical Physics, Vol. 2 (Pergamon, New York, 1987).

[23]G. 't Hooft, Nucl. Phys. B 190 (1981) 455; T. Suzuki et al., Prog. Theor. Phys. 80 (1988)

929; Phys. Lett. B 294 (1992) 100; H. Suganuma et al., Nucl. Phys. B 435 (1995) 207; see also M. Baker, J.S. Ball, and F. Zachariasen, Phys. Rep. 209 (1991) 73. 